\title{
Virtual-OCT: A simulated optical coherence tomography instrument
}

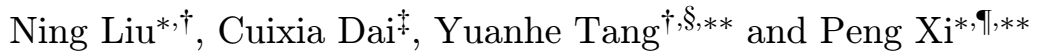 \\ *Department of Biomedical Engineering, College of Engineering \\ Peking University, Beijing 100871, P. R. China \\ $\dagger$ Department of Applied Physics, College of Science \\ Xi'an University of Technology, Xi'An 710048, P. R. China \\ *College of Science, Shanghai Institute of Technology \\ 100 Haiquan Road, Shanghai 201418, P. R. China \\ \$tangyuanhe@xaut.edu.cn \\ \xipeng@pku.edu.cn
}

Received 28 October 2013

Accepted 20 January 2014

Published 26 February 2014

\begin{abstract}
We report the virtual instrumentation of both time-domain (TD) and spectral-domain (SD) optical coherence tomography (OCT) systems. With a virtual partial coherence source from either a simulated or measured spectrum, the OCT signals of both A-scan and B-scan were demonstrated. The spectrometric detector's pixel number, dynamic range, noise, as well as spectral resolution can be simulated in the virtual spectral domain (SD-OCT). The virtual-OCT system provides an environment for parameter evaluation and algorithm optimization for experimental OCT instrumentation, and promotes the understanding of OCT imaging and signal post-processing processes.
\end{abstract}

Keywords: Optical coherence tomography; simulation; LabVIEW; depth-resolved imaging; crosssectional imaging.

\section{Introduction}

Optical coherence tomography (OCT) is an emerging noninvasive optical imaging method in clinical diagnosis. ${ }^{1}$ By combining low-coherence interferometry and confocal scanning microscopy, it can perform cross-sectional imaging of the internal microstructure of living tissue with an axial resolution of $1-15 \mu \mathrm{m}$ and penetration depth of $2-3 \mathrm{~mm} .^{2,3}$ It has been widely applied clinically in ophthalmology ${ }^{4,5}$ dermatology, ${ }^{6,7}$ gastroenterology, ${ }^{8,9}$ etc.

OCT utilizes a broadband, low temporal coherence light source as illumination. Based on the mechanism of signal detection and post-processing, OCT can be divided into two categories: (1) Timedomain OCT (TD-OCT), in which the depthresolved information is recorded though stepping

This is an Open Access article published by World Scientific Publishing Company. It is distributed under the terms of the Creative Commons Attribution 3.0 (CC-BY) License. Further distribution of this work is permitted, provided the original work is properly cited.

${ }^{* *}$ Corresponding authors. 


\section{N. Liu et al.}

the reference mirror, as partial interference can only be detected when the optical path difference is smaller than the coherence length. Because a mechanical scan is involved in each A-scan of TD-OCT, its applications are limited due to the slow imaging speed. (2) Fourier-domain OCT (FD-OCT), in which the depth-resolved structural information is extracted from the interference spectrum instead of moving the reference mirror. ${ }^{10}$ Taking advantage of the multiplexing detection (also known as Felgett's advantage), the imaging speed and sensitivity of FD-OCT are almost two orders of magnitude greater than for TD-OCT. ${ }^{11}$ FD-OCT can be subdivided into spectral domain OCT (SD-OCT) and swept source OCT (SSOCT) according to their detection methods. SDOCT employs a broad-bandwidth light source, and records the interference spectrum with a spectrometer ${ }^{11}$; SS-OCT, also known as optical frequency domain imaging (OFDI), uses a frequency-swept light source and a photoelectric detector, to record the interference spectrum as a function of time. ${ }^{12,13}$ The principle of SS-OCT is analogous to that of SD-OCT, although the interference spectrum is measured by different means.

Distinct studies have been performed on the simulation of OCT. The performance of OCT in highly scattering tissues has been simulated with the Monte Carlo method. ${ }^{11}$ Hosek et al. demonstrated the software control of a TD-OCT system, data acquisition and post-processing. ${ }^{14}$ Based on the simulation of SD-OCT detection, we reported on the optimization of SD-OCT with partial spectrum detection, pixel binning, etc. ${ }^{15}$ However, there have been no reports on the simulation of the partial interference process and post-processing of both kinds of OCT system. We constructed TD-OCT and SD-OCT systems by means of virtual instrumentation, and simulated the extraction of the depth-resolved information (A-scan) and the reconstruction of the cross-sectional image (B-scan). The virtual-OCT system should contribute to the research and development of OCT and optimization of the post-processing algorithm, as well as provides an intuitive and interactive platform for understanding the OCT processes.

\section{Computational Methods and Theory}

\subsection{TD-OCT theory}

TD-OCT is the first generation of OCT systems, in which the structure of the biological tissue is recovered by low-coherence Michelson interferometry with mechanical mirror scanning. Because the light source is a broadband low-coherence source, the interference occurs only within the coherence length. The interference signal represents the corresponding structural information of the specimen by scanning the reference arm. A schematic diagram of the TD-OCT system is shown in Fig. 1.

Using a Gaussian spectrum with power spectral density $S(k)$, central wavenumber $k_{0}$, and

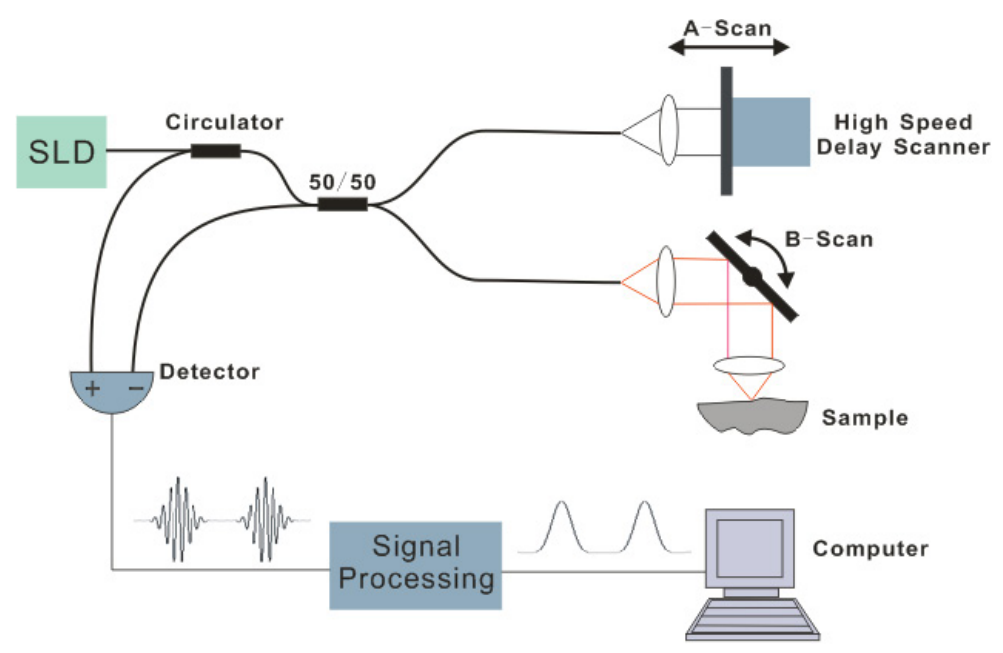

Fig. 1. Schematic diagram to illustrate the TD-OCT system. 


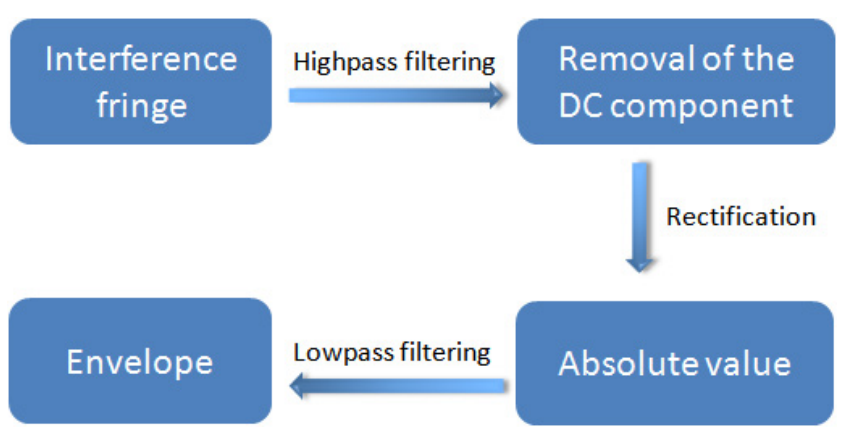

Fig. 2. Diagram of the TD-OCT post-processing algorithm.

wavenumber bandwidth $\Delta k$, the interference fringe $I_{D}\left(Z_{R}\right)$ is given $\mathrm{by}^{2}$ :

$$
\begin{aligned}
I_{D}\left(z_{R}\right)= & \frac{\rho}{4}\left[S_{0}\left[R_{R}+R_{S 1}+R_{S 2}+\cdots\right]\right] \\
& \text { DC component } \\
& +\frac{\rho}{2}\left[S_{0} \sum_{n=1}^{N} \sqrt{R_{R} R_{S n}} \mathrm{e}^{-\left(z_{R}-z_{S n}\right)^{2} \Delta k^{2}}\right. \\
& \left.\times \cos \left[2 k_{0}\left(z_{R}-z_{S n}\right)\right]\right]
\end{aligned}
$$$$
\text { Interference component. }
$$

Here, $S_{0}=\int_{0}^{\infty} S(k) \mathrm{d} k$ is the integrated power of the Gaussian spectrum, $R_{R}$ and $Z_{R}$ are the reflectivity and physical distance of the reference mirror respectively, $R_{S 1}, R_{S 2}, \ldots Z_{S 1}, Z_{S 2}, \ldots$ are the reflectivities and physical distances of each sample depth, respectively, and $\rho$ is the responsivity of the detector.
The first term in (1) is the DC offset and is proportional to the sum of the reference and sample reflectivities. The second term represents the interference of the reflected signal from the reference arm and the backscattered light from sample reflection of different depths, and the depth-resolved information lies in its envelope. Therefore, the post-processing of TD-OCT includes the steps shown in Fig. $2 .{ }^{16}$

\subsection{SD-OCT theory}

One major drawback of the TD-OCT system is that the mechanical scanning of the reference mirror limits the possible imaging speed, making it only applicable to situations of stationary imaging. To overcome this, a multiplex detection mechanism has been utilized in the SD-OCT. The reflected signal from the reference mirror simultaneously interferes with the backscattered light from each sample reflector. The depth-resolved structural information is acquired by taking the spectral-spatial transform of the interference spectrum measured by a spectrometer. The spectrometer is usually composed of a grating, a focusing lens and a line-CCD camera. SDOCT can achieve two orders of magnitude greater imaging speed due to the Felgett's advantage, therefore it is of great importance for demanding situations like retinal imaging. ${ }^{11}$ A schematic diagram of the SD-OCT system is illustrated in Fig. 3.

Using a Gaussian spectrum with a power spectral density $S(k)$, central wavenumber $k_{0}$ and

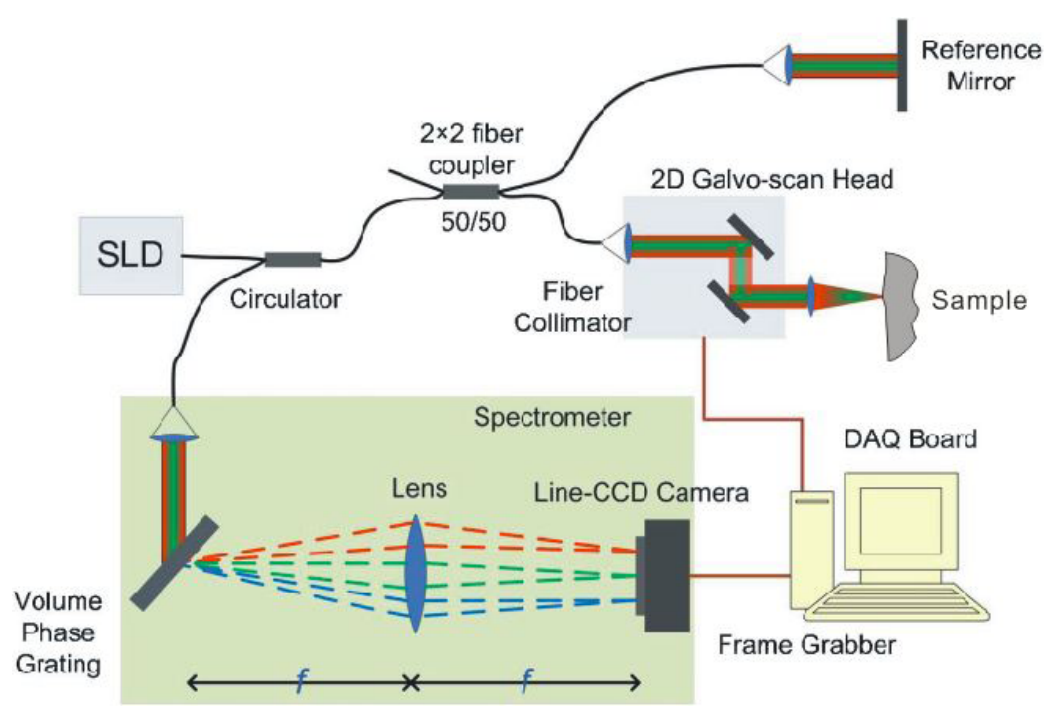

Fig. 3. Schematic diagram of SD-OCT system, which is simulated in our virtual instrumentation. 
wavenumber bandwidth $\Delta k$, the interference spectrum $I_{D}(k)$ is given $b^{2}$ :

$$
\begin{aligned}
I_{D}(k)= & \frac{\rho}{4}\left[S(k)\left[R_{R}+R_{S 1}+R_{S 2}+\cdots\right]\right] \\
& \text { "DC component" } \\
& +\frac{\rho}{2}\left[S(k) \sum_{n=1}^{N} \sqrt{R_{R} R_{S n}} \cos \left[2 k\left(z_{R}-z_{S n}\right)\right]\right] \\
& \text { Cross-correlation component } \\
& +\frac{\rho}{4}\left[S(k) \sum_{n \neq m=1}^{N} \sqrt{R_{S n} R_{S m}} \cos \left[2 k\left(z_{S n}-z_{S m}\right)\right]\right]
\end{aligned}
$$

Auto-correlation component.

The depth-resolved information can be obtained by taking the inverse fast Fourier transform (IFFT) of Eq. (2). The first term in (2) is often referred to as a "DC component" and is proportional to the sum of the reference and sample reflectivities (the first term varies with the variable $k$, not a real DC component). The second term is the cross-correlation of the signal from the sample arm and the beam from the reference arm, which is the desired signal for OCT imaging. The last term represents the auto-correlation of signals from sample reflection of different depths. Because the optical path length differences between the sample reflectors are typically small and the reflectivities of the sample reflectors are far less than that of the reference mirror, the auto-correlation component and $\frac{\rho}{4}\left[S(k)\left[R_{S 1}+R_{S 2}+\cdots\right]\right]$ can be omitted, but $\frac{\rho}{4}\left[S(k) R_{R}\right]$, which is often referred to as "auto-correlation of the light source spectrum" or "carrier envelope", is so large that it can overwhelm the desired signal. Therefore, the carrier envelope should be eliminated before taking the IFFT of (2), and a simple method is to record the interferogram with the reference mirror without a sample, then to subtract this signal component sequentially from the interference spectrum acquired in each A-scan.

The interference spectrum measured by a spectrometer is approximately mapped as a linear function of the wavelength $\lambda$, yet the IFFT relates the data evenly spaced in $k$-space. Therefore, the interference spectrum should be resampled in $k$-space before being transformed to the spatial domain, but resampling errors due to linear interpolation will be greater at greater physical distances. This error can be corrected using a fast Fourier transform (FFT)-zero padding-IFFT

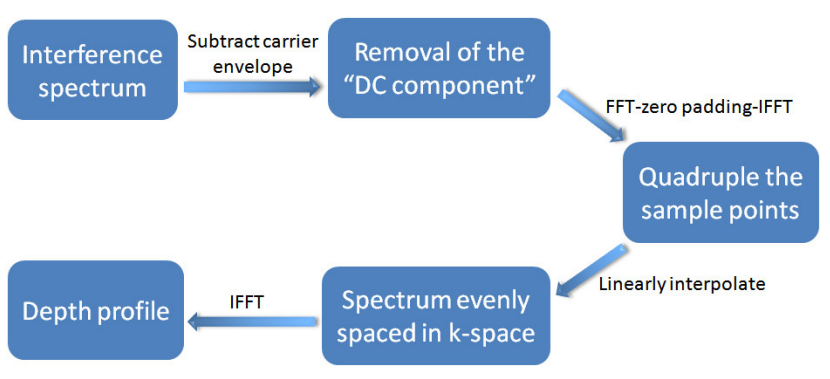

Fig. 4. Diagram of the SD-OCT post-processing algorithm.

algorithm, which increases the sampling points. Therefore, the post-processing of SD-OCT involves the steps shown in Fig. $4 .{ }^{11}$

\section{OCT Virtual Instrumentation}

\section{1. $S D-O C T$}

Based on LabVIEW virtual instrumentation, we simulated the process of the depth-resolved imaging and the cross-sectional imaging of the SD-OCT system. The depth-resolved information of the internal microstructures within the biological tissues can be obtained by the A-scan, and the cross-sectional image, or the tomogram can be acquired by the B-scan, which is composed of multiple A-scans.

\subsubsection{Depth-resolved imaging}

The flowchart of our virtual SD-OCT system for depth-resolved imaging is illustrated in Fig. 5. The functions of the six VIs in the flowchart are briefly described as follows:

- Gaussian_generation VI: Generates the theoretical Gaussian spectrum based on the input parameters (spectral width, spectral interval and central wavelength);

- Reflection VI: Calculates the complex amplitude of each wavelength composition reflected from the reference mirror or backscattered from each sample reflector;

- Noise_Generator VI: Generates the white noise signal (here for simplicity, we only simulated the white noise which is related with the dark current of the CCD camera);

- 2N_digitization VI: Digitizes the interference spectrum based on the dynamic range of the CCD;

- Frequency_Interpolate VI: Translates the spectrum evenly spaced in $\lambda$-space to that spaced as a linear function of $k$; 


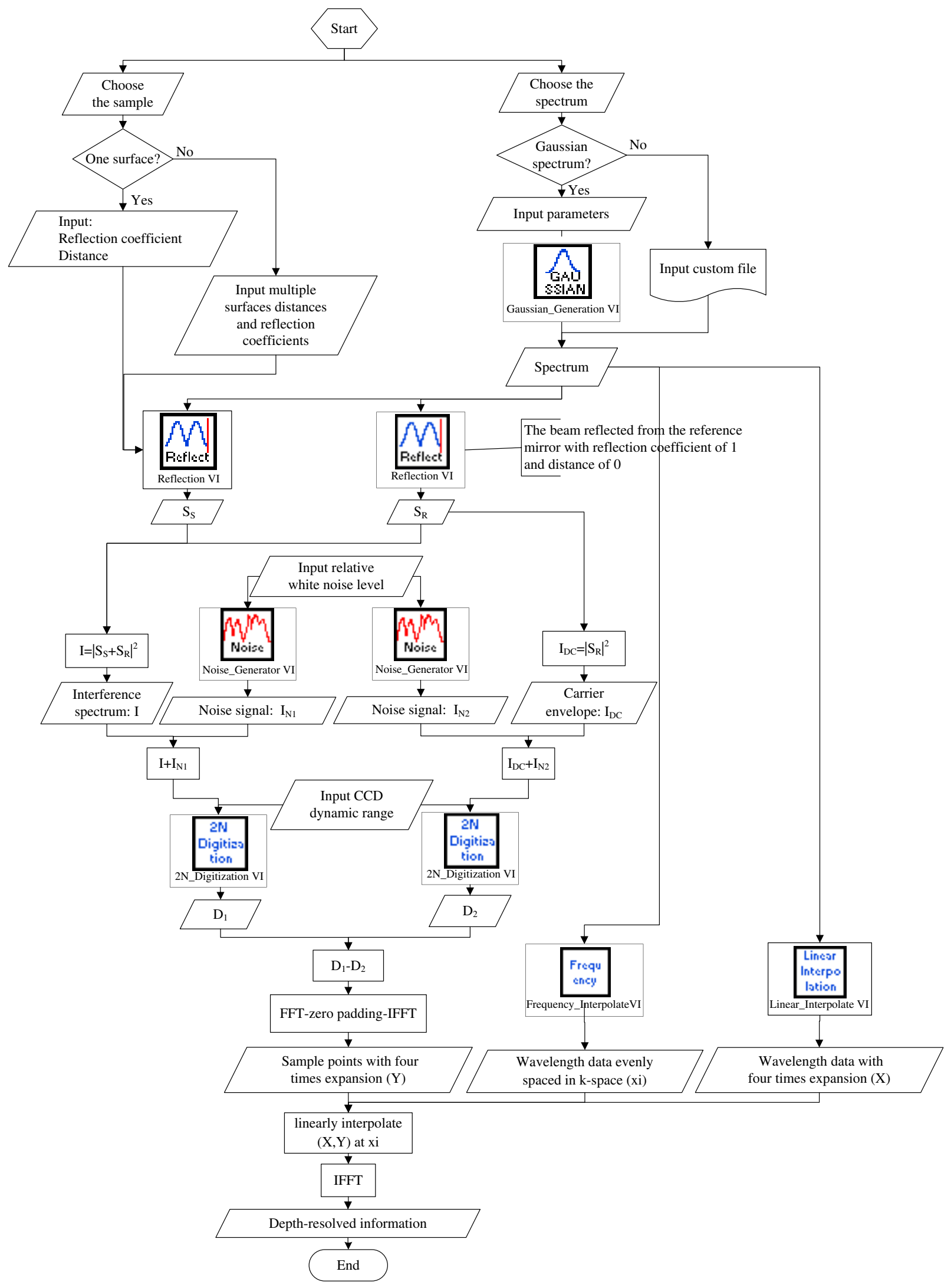

Fig. 5. The flowchart of the SD-OCT A-scan program. 
- Linear_Interpolate VI: Linearly interpolates the spectrum of the light source at the quarter points in order to quadruple the sample points;

Our SD-OCT A-scan program can be described in the following steps:

(1) The input spectrum and sample information. The spectrum of OCT light source can be input by generating a Gaussian distribution spectrum with Gaussian_generation VI, or input the custom spectrum file directly. Then, choose the sample comprised of one or multiple discrete surfaces and input the corresponding parameters (reflection coefficients at different distances).

(2) The interference. The essence of OCT process is the interference of the signal from the backscattering of the sample with the reference mirror reflection. Here, the complex amplitude backscattered from the sample $S_{S}$ was calculated by the Reflection VI taking the reflectivity information of the specimen, while the complex amplitude reflected from the reference mirror $S_{R}$ was obtained similarly (the reflection coefficient and distance of the reference mirror were assumed to be 1 and 0 for mirror reflection). The interference spectrum can be obtained by the squared modulus of their sum, and the carrier envelope was acquired by calculating the squared modulus of $S_{R}$.

(3) The noise evaluation. This is an optional function only for users to evaluate the real OCT signal with noise, such as dark current of the detector. To evaluate the effect of such kind of noise which is inevitable in a real OCT imaging process, here we have simulated a white noise signal with variable noise level. It is added to the carrier envelop as well as the interference spectrum. When the noise level is zero, it simulates an ideal OCT case. The users can replace the noise model with their own code to simulate different noise sources.

(4) Signal processing. First, the detected signal is digitized, then, to acquire the cross-correlation signal, the carrier envelope $D_{2}$ is subtracted from the interference signal $D_{1}$ to remove the "DC component". To increase the sampling rate, the cross-correlation signal was Fourier transformed to the spatial domain, then, zero padding is applied to quadruple the sample points, and inverse Fourier transformed back to the frequency domain with four times the points number labeled Y. Linear Interpolate VI linearly interpolated the spectrum of a light source at the quarter points to quadruple the sample points labeled X. Frequency_Interpolate VI translated the spectrum evenly spaced in $\lambda$-space to that spaced as a linear function of $k$ labeled xi. The interference spectrum evenly spaced in $k$-space was obtained by linearly interpolating $(X, Y)$ at xi. Finally, the data were inverse Fourier transformed to the depth space to obtain the depth-resolved information.

(5) Besides, the distance should be calibrated to reflect the actual physical size of the image to visualize the biological structure of the specimen, we achieved the calibration in the simulation using the relationship between the actual and sampling frequencies in a FFT algorithm. Assuming $\Delta k$ is the interval of the spectrum evenly spaced in $k$-space, $f_{s}$ is the sampling frequency of the spectrum. Based on the property of the FFT algorithm, the actual frequency (actual distance) of each depth-resolved datum

$$
f_{t}=\frac{f_{s}}{N} i=\frac{i}{\Delta k \cdot N},
$$

where $i$ is the index of the data and $N$ is the data size, thus the actual distances of the depth profile is calibrated. Grayscale image VI generated the grayscale image of the depth profile and the resolution of the OCT image can be measured with FWHM_calculation VI, which calculates the FWHM of the point-spread function (PSF) in the depth-resolved information.

\subsubsection{Cross-sectional imaging}

The cross-sectional OCT image (B-scan) consists of multiple depth-resolved images (A-scans). In SDOCT B-scan simulation, the grayscales of a grayscale image input by the user can be used as the reflection coefficients of the sample in different depths and positions. Here one can employ other image processing tools such as Image J, or further develop the program to apply color map on the image for better visualization of the biological structures.

As illustrated in Fig. 6, our SD-OCT B-scan program can be described in the following steps:

(1) Image to virtual sample information. Input a grayscale image with a known image resolution, Input image VI aimed to export its normalized grayscales, as the reflectivity is 8 bit, it was multiplied with $10^{-3}$ to convert grayscale to reflectivity 


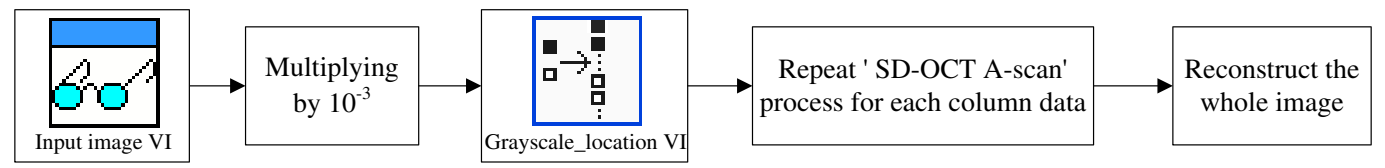

Fig. 6. The flowchart of the SD-OCT B-scan program.

for OCT imaging. The depth-resolved grayscales were transformed to multiple surface reflection coefficient of the sample by Grayscale_location VI. With a distance interval accuracy of $1 \mu \mathrm{m}$, the structure of the virtual sample is digitized for OCT process.

(2) Two-dimensional image reconstruction. Each column of data in the reflection coefficient array was processed with SD-OCT A-scan program to obtain the depth-resolved imaging (A-scan), and the B-scan was obtained with repeating the A-scan process of each position. Finally, the cross-sectional image was displayed.

\subsection{TD-OCT simulation}

TD-OCT is a traditional OCT with a straightforward mechanism and straightforward mathematical processing. As the imaging speed is limited by the mechanical scanning, TD-OCT is restricted to the speed insensitive applications. Here, we only simulated the depth-resolved imaging of the TD-OCT system. The flowchart is illustrated in Fig. 7.

TD-OCT differs from SD-OCT in two major respects: (1) The reference mirror is mechanically scanned to acquire the depth profile, so the distance of the reference mirror is an array determined by distance offset, sampling interval and sampling steps rather than a constant; (2) a photoelectric detector is used to record the interference intensity of all wavelength compositions as a function of depth.

Our TD-OCT A-scan program can be described in the following steps:

(1) The input spectrum and sample information. Choose the spectrum and the sample used in the simulation and input the corresponding parameters as well as the SD-OCT virtual instrument.

(2) The interference. Input distance offset, sampling interval and sampling steps to generate the distance of the reference mirror one by one with a "for loop", for each distance, the complex amplitude backscattered from the sample $S_{S}$ was calculated by the Reflection VI taking the reflectivity information of the specimen, while the complex amplitude reflected from the reference mirror $S_{R}[i]$ was obtained similarly (the reflection coefficient of the reference mirror was assumed to be 1 for mirror reflection). The interference intensity can be calculated by the formula $I[i]=\sum\left|S_{s}+S_{R}[i]\right|^{2}$. With the change of the reference mirror distance, the intensity array (i.e., the interference fringe) was obtained as a function of reference mirror distance.

(3) Signal processing. The intensity data was digitized and the DC offset was subtracted by removing its zero frequency, then, they were rectified by taking the absolute value, finally, the envelope (i.e., the depth-resolved information) was extracted by taking the maximum values and linearly interpolating them. Besides, the axial resolution of the simulated TD-OCT system was also given.

\subsection{Graphic user interface of the OCT virtual instrumentation}

Graphic user interface (GUI) plays a key role in virtual instrumentation. On one hand, the GUI is the only place where user can freely interact with the instrument, for both real and virtual ones. Therefore, the richness of input options and ease of use are two major parameters for industrial design. On the other hand, the intuitiveness for interaction is very important for users to grasp the main function quickly, without going to the complicated program (LabVIEW's back-panel). Taking advantage of the rich virtual instrumentation front panel GUI input icons, we have integrated both SD-OCT and TD-OCT in one virtual instrument, as illustrated in Fig. 8. Taking SD-OCT Ascan for example, the operations for the user are listed as follows: firstly, choose the spectrum used in the simulation (Gaussian or Custom spectrum). Once the illumination spectrum is chosen, the corresponding controls become visible, guiding the user to input the corresponding parameters such as Gaussian spectral width, Gaussian central 
N. Liu et al.

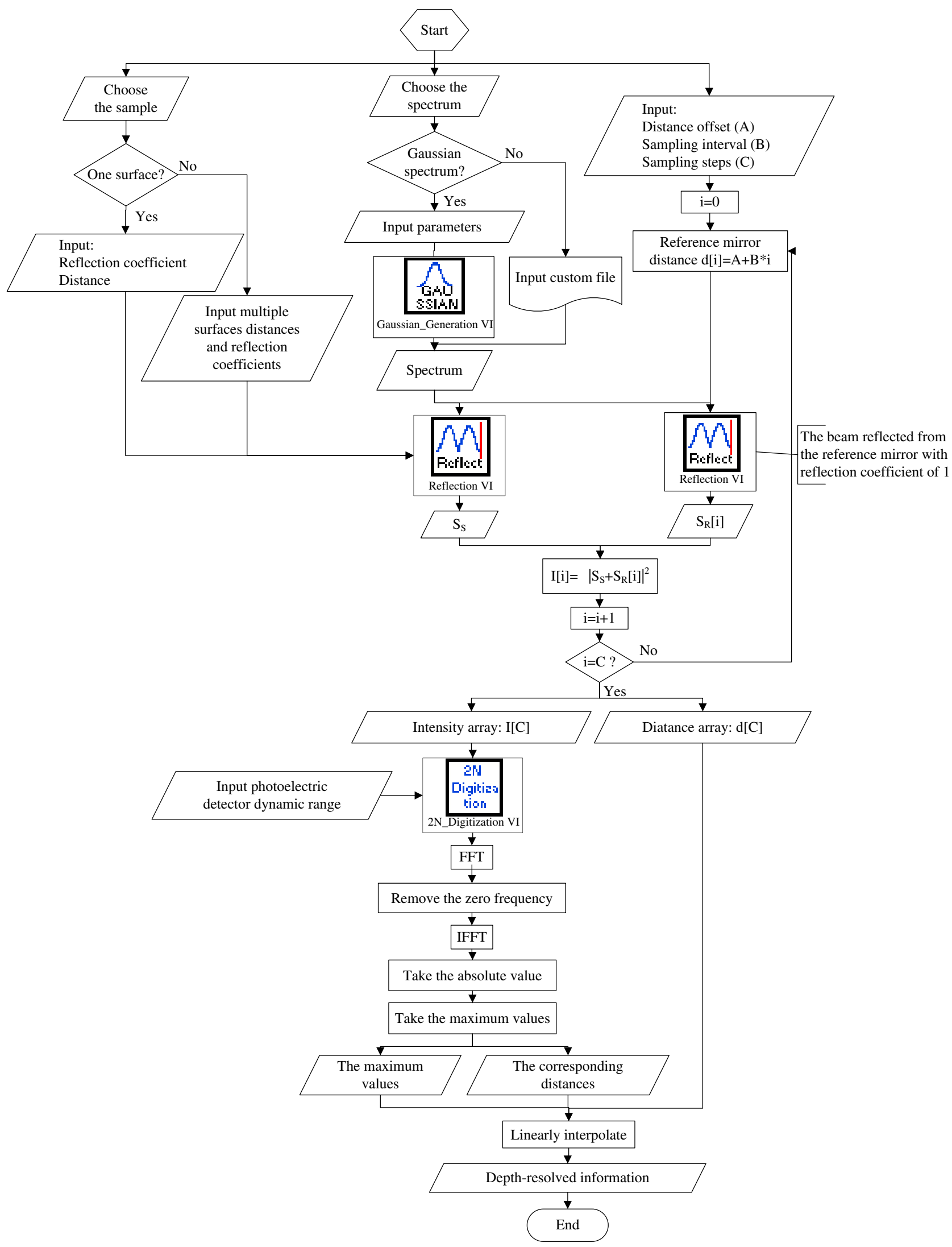

Fig. 7. The flowchart of the TD-OCT A-scan program. 


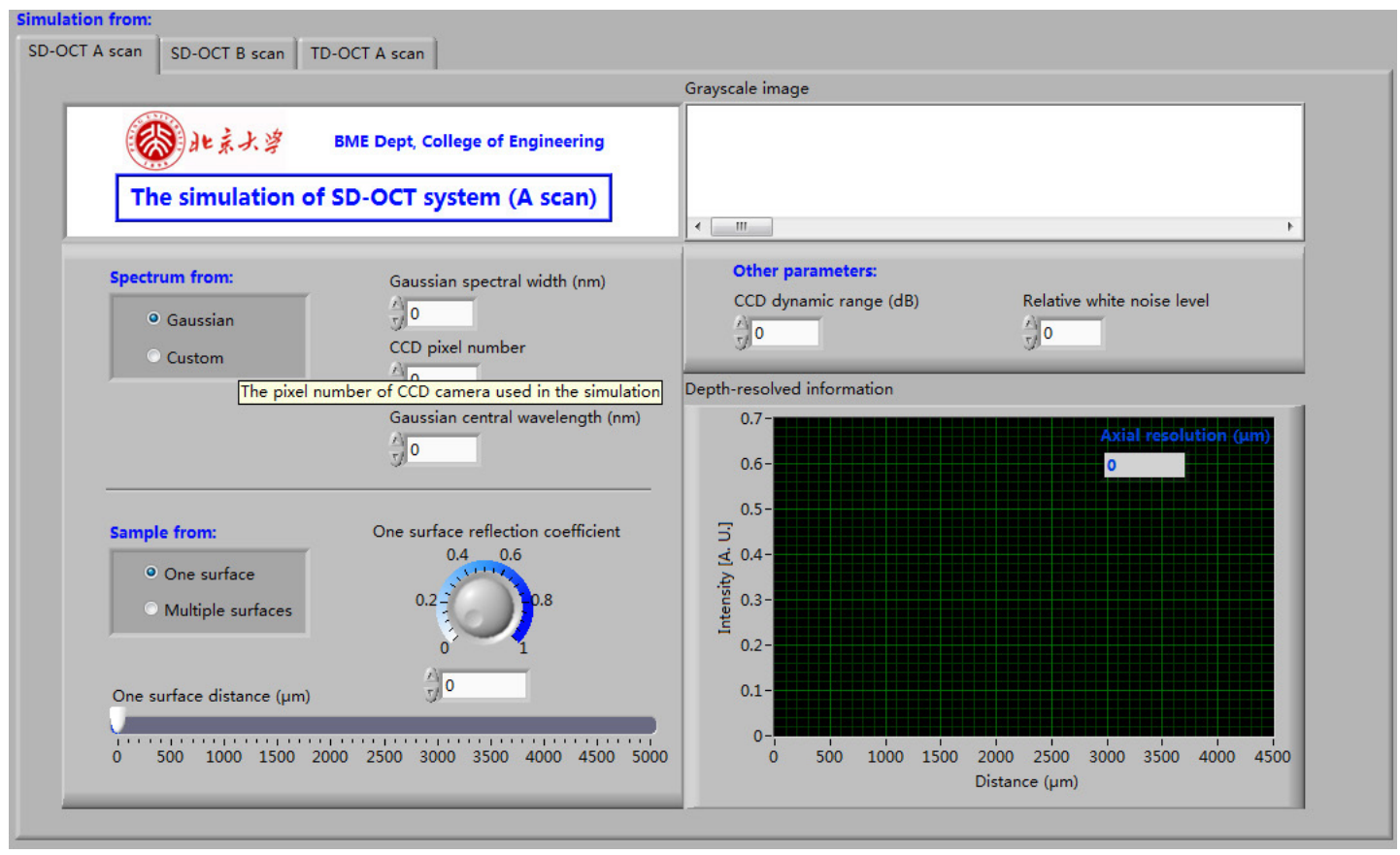

Fig. 8. GUI of the virtual OCT system. Note the yellow pop-up hint during input.

wavelength, etc. When the cursor is moved to a "control", its description is automatically displayed (Fig. 8) to help the user to better understand its function. Secondly, input the sample information which is comprised of one or multiple discrete surfaces. Finally, the OCT results, resolution, etc. are displayed automatically after the input.

\section{Results}

For an OCT system based on Gaussian spectrum, the axial resolution in air can be estimated by ${ }^{15}$

$$
\Delta z=\frac{2 \ln 2}{\pi} \frac{\lambda_{0}^{2}}{\Delta \lambda}
$$

Here, $\lambda_{0}$ and $\Delta \lambda$ denote the central wavelength and spectral width of the light source, respectively. The simulation results of a Gaussian spectrum are discussed in the paper.

\subsection{SD-OCT depth-resolved imaging}

With a Gaussian spectral width of $40 \mathrm{~nm}$, Gaussian central wavelength of $840 \mathrm{~nm}$, CCD pixel number of 2048, CCD dynamic range of $12 \mathrm{bits}$ and a relative white noise level of $0.5 \%$, the simulation result of sample 1 by the SD-OCT A-scan program is shown in Fig. 9 (the parameters of sample 1 are given in the upper right corner of the figure). From Fig. 9, one can see that, the OCT image reflects the physical structure of the simulated phantom accurately. The presence of mirror image in the simulated result is a typical artifact of SD-OCT.

\subsection{TD-OCT depth-resolved imaging}

Assuming the distance offset, sampling interval and sampling steps to be $10 \mu \mathrm{m}, 0.01 \mu \mathrm{m}$ and 8000 , respectively, so that the reference mirror was simulated to move from 10 to $90 \mu \mathrm{m}$ in 8000 steps. With a Gaussian spectral width of $40 \mathrm{~nm}$, Gaussian

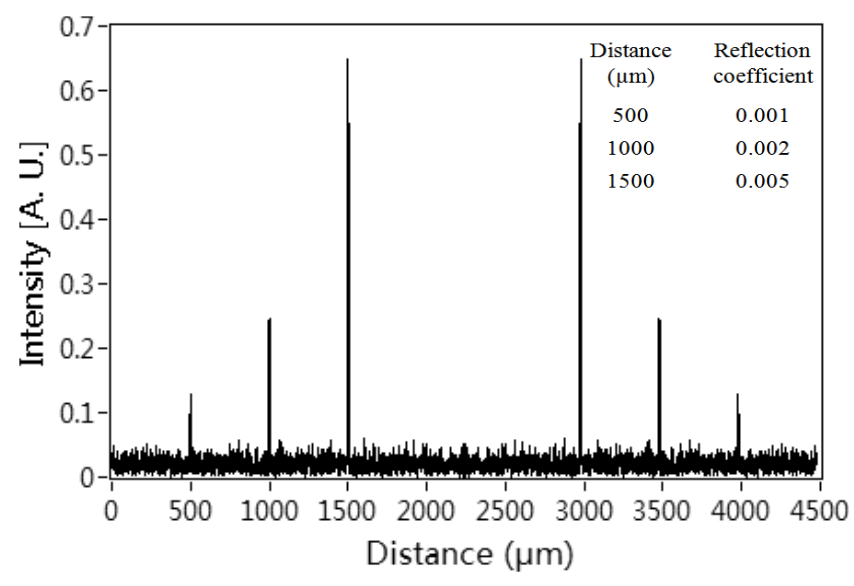

Fig. 9. The simulation result of sample 1 by the SD-OCT A-scan program. 
central wavelength of $840 \mathrm{~nm}$, Gaussian spectral interval of $1 \mathrm{~nm}$ and a detector dynamic range of 12 bits, the simulation results of sample 2 by the TD-OCT A-scan program are illustrated in Fig. 10 [the parameters of sample 2 are given in the upper left corner of Fig. 10(b)]. In the TD-OCT simulation, (a) was the interference fringe recorded by the photoelectric detector; (b) was acquired by extracting the envelope of (a).

As a comparison with the theory, we simulated the relationship between the axial resolution and Gaussian spectral width of the simulated SD- and TD-OCT systems with Gaussian central wavelength of 840,1300 and $1550 \mathrm{~nm}$ (the central wavelengths of SLD commonly used in OCT systems), respectively. The simulation results and the theoretical values are shown in Fig. 11, from which one can see that the simulated axial resolutions show good agreement with the theoretical values for each Gaussian central wavelength. This demonstrated the validity of the algorithms used in our simulation and illustrated that the virtual OCT system can be used to evaluate and optimize the axial resolution of a SD- or TD-OCT systems.

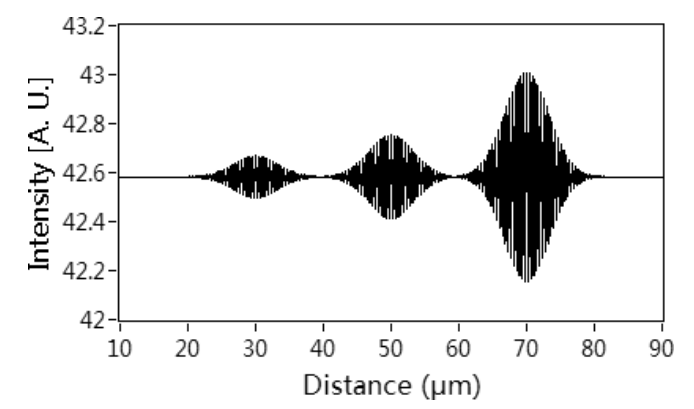

(a)

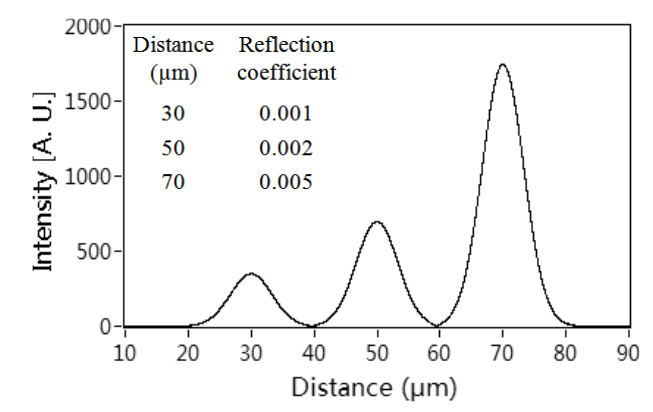

(b)

Fig. 10. The simulation result of sample 2 by the TD-OCT Ascan program. (a) The interference fringe. (b) The post-processed OCT image reflects the depth-resolved structural information of the simulated phantom.

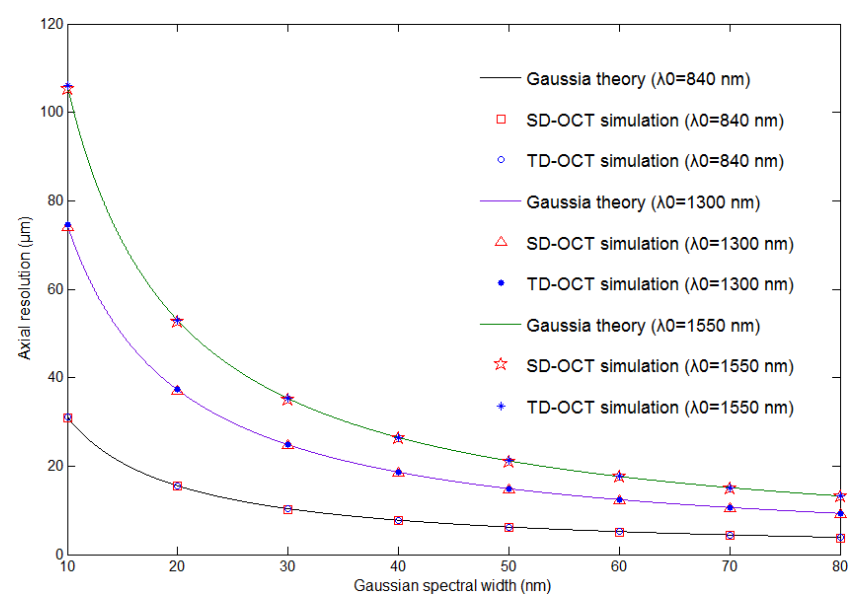

Fig. 11. The axial resolution of the simulated SD- and TDOCT systems.

\subsection{SD-OCT cross-sectional imaging}

OCT has been applied onto the detection of a variety of cancers. ${ }^{17}$ However, it should be noted that the $5-10 \mu \mathrm{m}$ resolution of a typical OCT system is insufficient for imaging of individual cells. Therefore, the OCT cancer detection can only be limited to those with large morphological changes, rather than the subtle cellular level indication. To validate the usefulness of an OCT system in cancer detection, we have constructed the OCT for cross-sectional imaging, in which one can input a histological image as a target for OCT imaging, and generate the corresponding OCT result to evaluate whether such OCT system can be applied on this cancer detection noninvasively or not. Here, a retinal histology image [Fig. 12(a)] with a resolution of $7 \mu \mathrm{m} /$ pixel was used as input for the SD-OCT B-scan program. With a Gaussian central wavelength of $840 \mathrm{~nm}$, CCD pixel number of 2048, CCD dynamic range of $12 \mathrm{~dB}$ and a distance offset of $10 \mu \mathrm{m}$, the reconstructed images with different OCT axial resolutions are shown in Figs. 12(b)-12(d). The OCT axial resolution is determined by the Gaussian spectral width, in the case of the same Gaussian central wavelength. As can be seen, with the lower OCT axial resolution, the reconstructed images become further blurred. These results facilitate the physicians to evaluate the application and limitation of OCT system in its cancer detection.

In this work, only the interference between the sample arm and the reference arm is considered. It should be noted that, other factors such as scattering (which is the dominant factor for the imaging 


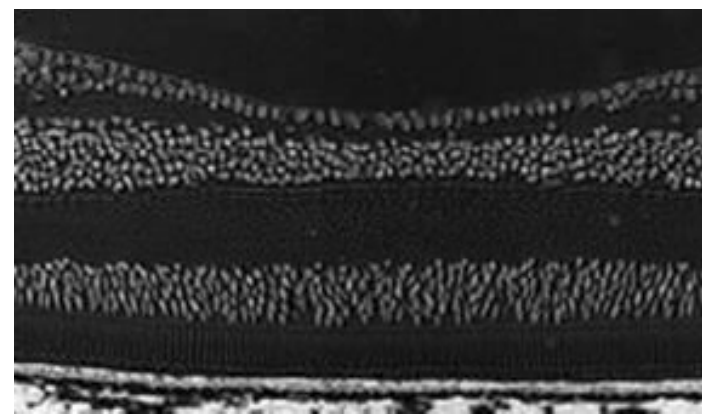

(a)

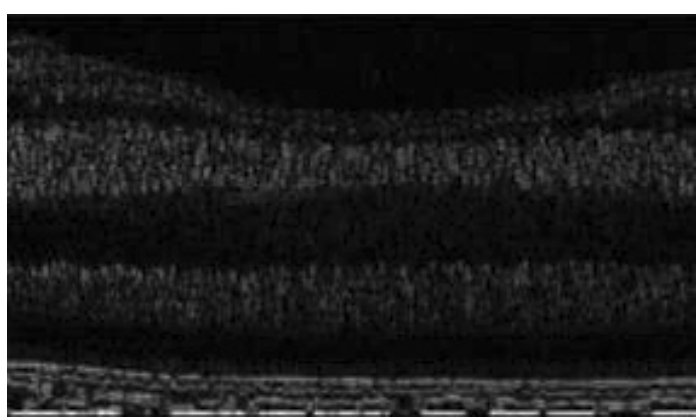

(c)

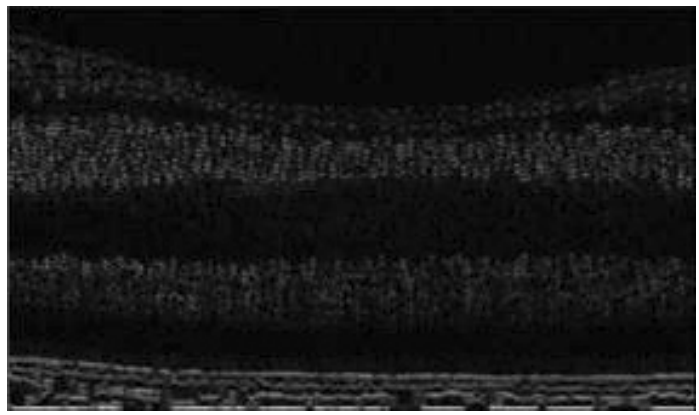

(b)

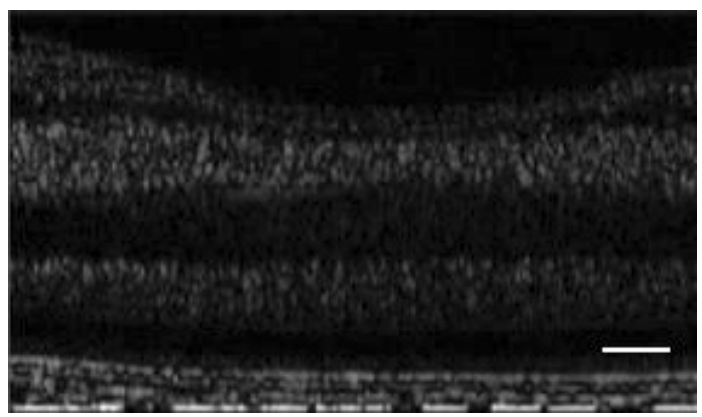

(d)

Fig. 12. (a) The input retinal image. (http://www.iupui.edu/ anatd502/Labs.f04/eye\%20lab/pages/s94_ 10x_ 15_jpg.htm). (b), (c), (d) are the reconstructed images with a OCT axial resolution of 5, 10 and $20 \mu \mathrm{m}$, respectively. Scale bar: $200 \mu \mathrm{m}$.

depth of OCT) and absorption (especially when the blood vessels are presented) should be considered to further simulate the situation of OCT imaging for biological specimen. The effect of the scattering to the sample signal intensity can be simulated as one or multiple exponential decays across the propagation distance, following the Beer-Lambert law. ${ }^{16}$ The effect of the absorption can be simulated similarly, with some abrupt change of absorption coefficient assigned for sites such as the blood vessel.

\section{Conclusion}

We have constructed the virtual-OCT system for both time-domain (TD) and spectral-domain (SD) imaging. The depth-resolved imaging, crosssectional imaging of the SD-OCT system, as well as the depth-resolved imaging of a traditional TD-OCT system were simulated. The axial resolutions of the simulated TD- and SD-OCT systems are in agreement with the theoretical values, and their depth-resolved information well recovers the sample structure. With the input of a histological image which represents the structure of the tissue being examined, the cross-sectional image from the virtual OCT can be used to help evaluating the applicability of the real OCT system in cancer detection. The virtual-OCT system provides an intuitive and interactive platform for understanding OCT processes, and an environment for parameter evaluation and algorithm optimization, which can contribute to the research and development of the next generation OCT system.

\section{Conflict of interest statement}

The authors claim no conflict of interest.

\section{Acknowledgments}

The authors thank Dr. Thomas FitzGibbon for comments on earlier drafts of the manuscript. This research is supported by the National Instrumentation Program (2013YQ03065102), the "973" Major State Basic Research Development Program of China (2011CB707502, 2010CB933901, 2011CB809101), and the National Natural Science Foundation of China (61178076, 61307015). Ning Liu and Cuixia Dai equally contributed to this paper. 


\section{Appendix}

The virtual-OCT system used in this work can be downloaded from: http://bme.pku.edu.cn/ xipeng/ Tools.htm.

\section{References}

1. D. Huang et al., "Optical coherence tomography," Science 254(5035), 1178 (1991).

2. W. Drexler, Optical Coherence Tomography: Technology and Applications, Springer Verlag (2008).

3. J. G. Fujimoto et al., "Optical coherence tomography: An emerging technology for biomedical imaging and optical biopsy," Neoplasia, New York, NY, 2(1-2), 9 (2000).

4. A. Fercher et al., "In vivo optical coherence tomography," Am. J. Ophthalmol. 116(1), 113 (1993).

5. E. A. Swanson et al., "In vivo retinal imaging by optical coherence tomography," Opt. Lett. 18(21), 1864-1866 (1993).

6. J. Welzel, "Optical coherence tomography in dermatology: A review," Skin Res. Technol. 7(1), 1-9 (2001).

7. M. C. Pierce et al., "Advances in optical coherence tomography imaging for dermatology," J. Invest. Dermatol. 123(3), 458-463 (2004).

8. G. Tearney et al., "Optical biopsy in human gastrointestinal tissue using optical coherence tomography," Am. J. Gastroenterol. 92(10), 1800 (1997).
9. J. A. Izatt et al., "Optical coherence tomography and microscopy in gastrointestinal tissues," Sele. Top. Quantum Electron. [IEEE] 2(4), 1017-1028 (1996).

10. A. F. Fercher et al., "Measurement of intraocular distances by backscattering spectral interferometry," Opt. Commun. 117(1-2), 43-48 (1995).

11. M. Wojtkowski et al., "In vivo human retinal imaging by Fourier domain optical coherence tomography," J. Biomed. Opt. 7, 457 (2002).

12. S. Chinn, E. Swanson, J. Fujimoto, "Optical coherence tomography using a frequency-tunable optical source," Opt. Lett. 22(5), 340-342 (1997).

13. B. Golubovic et al., "Optical frequency-domain reflectometry using rapid wavelength tuning of a Cr4+: Forsterite laser," Opt. Lett. 22(22), 17041706 (1997).

14. P. Hosek et al., "Application of LabVIEW: Complex software controlling of system for optical coherence tomography," J. Assoc. Lab. Automation 14(2), 5968 (2009).

15. P. Xi et al., "Evaluation of spectrometric parameters in spectral-domain optical coherence tomography," Appl. Opt. 50(3), 366-372 (2011).

16. L. V. Wang, H. Wu, Biomedical Optics: Principles and Imaging, Wiley-Blackwell (2007).

17. A. Sergeev et al., "In vivo endoscopic OCT imaging of precancer and cancer states of human mucosa," Opt. Express 1(13), 432-440 (1997). 